\title{
Dietary and anthropometric factors affecting the potential of gut microbiota to utilize dietary fiber and produce short-chain fatty acids
}

\author{
$\underline{\text { Anna M. Malinowska }}^{1}$, Marcin Schmidt ${ }^{2}$, Malgorzata Majcher ${ }^{3}$, Hanna Przydatek ${ }^{1}$, \\ Marta Szaban ${ }^{1}$ and Agata Kurowiecka ${ }^{1}$ \\ ${ }^{1}$ Poznańn University of Life Sciences/Institute of Human Nutrition and Dietetics, Poznań, Poland, \\ ${ }^{2}$ Poznań University of Life Sciences/Department of Biotechnology and Food Microbiology, Poznań, Poland and \\ ${ }^{3}$ Poznan University of Life Sciences/Institute of Food Technology of Plant Origin, Poznan, Poland
}

\section{Abstract}

Some species of gut bacteria produce short-chain fatty acids (SCFAs) from dietary fiber-mainly acetate, propionate, and butyrate. The composition of human gut microbiota is dependent on dietary intake and health status. The aim of this study was to assess the effect of diet and anthropometric parameters on the potential of gut microbiota to metabolize dietary fiber and produce SCFA.

A group of 200 men and women aged 31 to 50 years old participated in the study. The diet was assessed using three-day dietary records and the dietary pattern was determined using score methods. The potential to utilize water-insoluble fiber was assessed by measuring the $\beta$-glucosidase enzymatic activity of dissolved feces. To estimate the potential to metabolize water-soluble dietary fiber, cultures containing feces and pectin were incubated under anaerobic conditions for 24 hours. The amounts of fiber, acetic acid, propionic acid, and butyric acid before and after incubation were measured.

Pectin utilization correlated positively with the amount of energy intake from fat $(r=0.19)$ and with the intake of nuts and seeds $(r$ $=0.17)$ and was negatively correlated with the amount of energy from complex carbohydrates $(r=-0.16)$ and its sources, such as refined grain products $(\mathrm{r}=-0.15)$. The dietary pattern did not affect the potential of the gut microbiota to metabolize pectin, but did influence the potential to digest insoluble dietary fiber, as the subjects following the western dietary pattern had lower potential than those following the rational pattern. $\beta$-glucosidase activity correlated positively with the intake of dietary fiber $(r=0.19)$ and intake of its sources, such as fruits $(r=0.18)$, vegetables $(r=0.21)$, and nuts and seeds $(r=0.18)$; it correlated negatively with nonalcoholic beverage intake $(r=-0.15)$ and sugar and honey intake $(r=-0.16)$. The potential to synthesize acetic acid correlated negatively with dietary indices and dietary fiber intake $(\mathrm{r}=-0.18)$. The potential to synthesize propionic acid correlated negatively with hip and waist circumference $(r=-0.14,-0.15$, respectively). The potentials to synthesize both propionic and butyric acid were affected by the intake of nuts and seeds ( $\mathrm{r}=0.18,0.21$, respectively).

Diet affects the potential of gut microbiota to utilize dietary fiber and to produce SCFAs. The impact of anthropometry parameters was only seen on the potential to synthesize propionic acid.

\section{Funding}

National Science Centre, Poland (2015/17/D/NZ9/01959)

\section{Conflict of Interest}

There is no conflict of interest 